\begin{tabular}{|c|c|c|}
\hline Received 16.10.2020 & \multirow{3}{*}{ Research Article } & \multirow{3}{*}{$\begin{array}{c}\text { JOTS } \\
5 / 1 \\
\text { 2021: } 195-204\end{array}$} \\
\hline Accepted 3010.2020 & & \\
\hline Published 10.01.2021 & & \\
\hline
\end{tabular}

\title{
Nachträge zu "Die Lehre des Buddha und das Königshaus des Westuigurischen Reichs: Die vier Begegnungen"
}

\author{
Ek: “Buddha'nın Öğretisi ve Batı Uygur Kağanlığı Hanedan: Dört Karşılaşma”
}

\author{
Peter ZIEME* \\ Berlin/Germany \\ E-mail:ziemepet@gmail.com
}

By mistake two text portions are missing in the paper published in JOTS 2020. As AppA and AppB they are here edited as additions. The word forms of these two fragments were already included in the index of the main article.

Key Words: Buddhism, Four Encounters of the Legendary Buddha Biography, Western Uighur Royal Family, Old Uighur language, SI 5671 (2Kr 49), U 1824 (T II T 103).

ORCID ID: 0000-0002-8090-7707. 


\section{ग(৫)}

Durch ein Versehen sind zwei Texte, die als Anhänge (AppA und AppB) gedacht waren, aus meinem Beitrag in letzten Heft des JOTS (4/2, 2020: 546-731) ausgefallen, obwohl die Wortformen sogar im Index ${ }^{1}$ enthalten sind. Hier sollen diese beiden Fragmente als AppA und AppB nachgeliefert werden.

\section{AppA}

SI 5671 (2Kr 49). Eine weitere Handschrift, es handelt sich um ein beidseitig beschriebenes Blatt aus einem gehefteten Buch, scheint in diesen Kreis zu gehören, wenngleich die für die anderen Fragmente ermittelte Struktur nicht erkennbar wird und auch jegliche übereinstimmende Parallelen fehlen. Der Text dieses Bruchstücks läßt sich am besten dem Thema ,Tod' zuordnen.

Als Besonderheit der Schrift ist zu verzeichnen, daß fast kein Unterschied zwischen den medialen Formen der Buchstaben $\langle>>,<\mathrm{n}>$ und $<\mathrm{r}>$ besteht.

Das bisher unbekannte Wort intravar hatte W. Radloff bereits in sein Uigurisches Wörterbuch neben weiteren aus damals unpublizierten Texten aufgenommen: ermpaвap ,die blaue Perle‘ (2 Kr.49,3) .

\section{Recto}

01 ol kim tört türlüg ärdnin yaratmı̌̌

02 ol . bir yüzi altun . ikinti yüzi kümüš .

03 üčünč yüzi intravar . törtünč yüzi $p(a) d m a$

$04-$ rog $^{3}$. kün ay t(ä)yri ordusinta tägzinür .

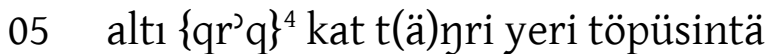

06 turur . üdi k(ä)lsär tüšär yimrilür . bir kog

07 ’ävininčä kalmaz . tört taluy ögüz suvı

\footnotetext{
1 Durch die erneute Kontrolle der Lesungen entfallen die im Index verzeichneten Belege für yägän und tarkar-.

2 Radloff, 1917: Sp. 198. In Sp. 103 zitiert aus demselben Fragment auch die Namen von Z. 18 (unter am arčuni).

3 pdm'-rwk.

4 Getilgtes Wort.
} 
08 ol sugulur bir tamaz kalmaz . č(a)hšapt tut

09 -dačı bilgälär-kä enč ıdmaz . ap bay ap čıay

10

$[\ldots] \mathrm{d}[\ldots . . . . ..] \mathrm{p}[\quad] \mathrm{pumaz}$.

\section{Verso}

11[

12 barčanı yok yodun k1lur . antag yüzsüz

13 tavar ol bo ölüm . bo yertinčü yer suv äy

14 bašlayu b(ä)lgürmiš-tä m(a)ha-sampadi atl(1)g

15 elig han b(ä)lgürdi . sans(1)z sakıšsız yılın ayın

16 bo yertinčü-dä elänti . ölüm küni [k](ä)lti ol

17 elänmäk ärksinmäk näy tusulmatı . ölüm

18 eyin bard1-lar . rami arčuni atl(1)g tona yaya

19 bar ärti-lär . üküš üdün öčkä yarıška

20 kirdilär kamag yegäddi ${ }^{5}$ uttılar : k(ä)ntünün

\section{Übersetzung}

\section{Recto}

[Der Sumeru ist es], der versehen ist mit den viererlei Juwelen ${ }^{6}$. Eine Seite ${ }^{7}$ Gold, die zweite Seite Silber, die dritte Seite indravar ${ }^{8}$, die vierte Seite padmarog ${ }^{9}$.

Geschrieben: $y y k^{2} t d y$.

6 Eine genaue Parallele für die hier gebrauchte Reihe ist nicht nachzuweisen. Allgemein ist die Viererreihe Gold, Silber, Lapislazuli (vaidūrya), Sphațika, vgl. Zieme, 2020: 161. Anders die Reihe der 7 Juwelen, die zum einen eine 7 reale Juwelen umfaßt, zum anderen die 7 königlichen Schätze, die dem Cakravartinrāja eigen sind. (Soothill \& Lodous, 1937: 12b-13a).

$7 y$ yü, Gesicht', aber auch ,Seite‘ etc.

8 Die hier bezeugte Form intravar ließe sich am ehesten auf skt. *indravara zurückführen, doch belegt ist nach Monier-Williams, 1899: 166a indivara ,the blossom of a blue lotus', so dürfte hier eine Kreuzung aus indra und vāra vorliegen.

9 Dabei handelt es sich wahrscheinlich um eine Übernahme aus skt. padmarāga ,lotus-hyed, ruby' (Monier-Williams, 1899: 584c), doch die Schreibung rwk läßt eher an skt. roga (< Vruj) denken. Die Frage ist, ob es skt. *padmaroga als eine Juwelfarbbezeichnung gibt. Vgl. Wojtilla, 1973: 218. 
Mit Sonne und Mond ${ }^{10}$ wandelt er (?). ${ }^{11}$ Das sechsstufige Himmelsland ${ }^{12}$ ist auf seiner Spitze. Wenn die Zeit kommt, fällt er und bricht zusammen, nicht ein Staubkörnchen bleibt. Das Wasser der vier Meere, es verschwindet, nicht ein Tropfen bleibt. Den Weisen, die die Gebote halten, schickt er ${ }^{13}$ keinen Frieden. Reiche wie Arme [trifft die Vernichtung (?),] kann nicht [ ].

\section{Verso}

[ ] alles vernichtet ${ }^{14} \mathrm{er}$. So eine unverschämte ${ }^{15}$ Sache ist er, dieser Tod. Nachdem die irdische Welt zuerst entstanden ist, ist der Mahāsaṃmata ${ }^{16}$ genannte König erschienen. In zahllosen Jahren und Monaten hat er auf dieser Welt geherrscht. Der Tag des Todes kam. Das Herrschen und Mächtigsein hat nicht genutzt. Dem Tod folgend ist er dahingeschieden ${ }^{17}$. Die Helden ${ }^{18}$ Rāma $^{19}$, Arjun $^{20}$ gab es. Viele Male traten sie in Kämpfe und Wettkämpfe ein. Immer siegreich waren sie und gewannen. Selbst [am Ende doch starben sie.]

10 Wörtlich: ,in dem Palast von Sonne und Mond".

11 Kirfel 1920, 183 nach AN: „Soweit ... wie Sonne und Mond umherwandeln und die Weltgegenden mit ihrem Schein erleuchten, soweit ist eine tausendfache Welt.“

12 六天 ,The six devalokas ... above sumeru, between brahmalokas and earth“ (Soothill \& Lodous, 1937: 134a).

13 Dem Zusammenhang nach ist hier wohl der ,Tod‘ zu verstehen.

14 In Maitr Ham XV 6b heißt es vom ,Alter' (karımak): ät’özdäki küč küsünüg osar köyüldäki y(i)ti katıglanmak tülüklänmäkig koratur sävig köyül akrušlanmak körtlä körküg yigit türk ät’özüg kaltı tolı tokımıš lenhua čäčäklig arag osuglug yok yodun kllur "(Es) bricht die Kraft des Körpers, mindert die scharfe Bemühenskraft des Sinnes und macht die durch Liebe (entstandene) ruhige und schöne Gestalt und den jugendlich-starken Körper zunichte, wie einen Wald von Lotosblüten, der vom Hagel getroffen ist."

15 Wörtlich, gesichtslos‘, vgl. so auch ttü. yüzsüz, frech, unverschämt‘.

16 Wilkens, 2016: 00484. Der Name ist hier ergänzt zu m(a)[sama]țe. Angesichts des anderen altuigurischen Belegs in einem buddhistischen Kolophongedicht als maha-sanbade (Gabain, 1967: 2022) sowie der darauf basierenden mongolischen Formen Maqasabadi oder Maqasambadi (Kara, 2009: 176) stellt sich die Frage, ob entsprechend auch der DKPAM-Eintrag so gelesen werden sollte.

17 Pluralis majestatis. Allerdings könnte sich die Verbform auch auf alle Personen beziehen.

18 Wie auch an anderen Stellen wird hier für ,Helden“ das Kompositum toya yaya ,Leoparden und Elefanten" verwendet.

19 Wilkens, 2016: 00472 etc.

20 Wilkens, 2016: 00501 etc. 


\section{J(e)}

\section{Kommentar}

Hier könnte der Sumeru gemeint sein, dessen Seiten aus Gold, Silber, Beryll und Kristall bestehen ${ }^{21}$. Über dem Sumeru ziehen Sonne und Mond ihre Bahnen. ${ }^{22}$ Die sechsfache Götterwelt (kāmadhātu ${ }^{23}$ steht auf dem ,Scheitel'. Doch wenn die Zeit kommt, bricht alles zusammen, so wie es auch im ersten der 5 schlechten Träume heißt ${ }^{24}$.

\section{AppB}

"Könige oder Fürsten, die meine Disziplin einführen, werden mein LehrLeben verlängern!" So heißt es in dem Fragment U 1824 (T II T 103). Es handelt sich um ein einzelnes Blatt aus einer Pustaka-Handschrift, deren Bestimmung noch aussteht. Falls die Handschrift ein Sūtratext war, könnte man an einen ähnlichen Wortlaut denken, wie er im 12. Kapitel des Goldglanz-Sūtras vorliegt. In der Beschützung des Landes durch die vier Himmelskönige preist der Buddha diejenigen, die dem Sūtra Verehrung erweisen, allen voran die vier Himmelskönige. ${ }^{25}$ Das 20. Kapitel behandelt das Verhalten der irdischen Könige gemäß dem rājaśāstra. Aber im folgenden Text geht es ganz direkt um irdische Könige, die für den Fortbestand der Lehre sorgen. ${ }^{26}$ Ähnliches findet man u. a. im Sūtra Prajñāpāramitā - Menschliche Könige beschützen das Land (T 245/246 人王護 國般若波羅密多經), wo es heißt:

"Thus the role of kings is to be protectors and guardians-or mothers-of the Samgha. At the same time the practices characteristic of kingship-particularly the protection of the Teaching-lead to the acquisition of Buddhist lineage (hsi-chung-hsing) and realization of one's innate Buddhahood (hsing-chung-ching) and thus to full deployment of the Way (the

21 Meier, 1988: 570.

22 Meier, 1988: 709. „Sonne und Mond mit Sternbildern haben ihren Platz links und rechts der am tiefsten eingeschnittenen Stelle des Weltberges".

23 Meier, 1988: 539. Inagaki, 1994: 382.

24 T.XII.0383.1012a19-20 於其夜得五大惡夢. 一夢須彌山崩四海水竭. “In dieser Nacht hatte sie fünf große schlechte Träume. Der erste Traum handelte vom Zusammenbrechen des Sumeru-Bergs und vom Austrocknen der vier Ozeane."

25 Nobel, 1958: 89. „Wenn ihr dieses Sūtra beschützt und festhaltet, dann werdet ihr durch die Kraft des Sūtra die vielen Nöte, die Widersacher, die Hungersnöte und Seuchen beseitigen. Wenn ihr daher seht, dass die vier Gemeinden diesen Sūtra-König annehmen, festhalten, lesen und rezitieren, dann sollt ihr auch eifrigen Sinnes sie sorgsam beschützen, ihre Bedrängnisse beseitigen und ihnen Wohlergehen gewähren."

26 Im Gegensatz zu den ,schlechten Königen“ in der Zeit des Niedergangs (魔法 mofa), vgl. Nattier 1991. 


\section{J(O)}

practices and progress of the bodhisattva, tao-chung-hsing). The scheme of the five forbearances sets out a continuous progress from king through Buddha, and through it effectively erases the taxonomic line between bodhisattvas and kings." ${ }^{27}$

\section{Text}

\section{Recto (?) $)^{28}$}

$01 \quad$ [ ]-1g yitd[i]m[iz

02 [täyri täyris]i burhan-nıy nomlug y[arlıgın]

03 [äšidmäktä] yeg ärmäz : tutar-s[iz]

04 [ ] kayu yer-kä tayıp

05 [ ] yer-kä ta[ya]nıp turmı̌̌

06 [ ] ymä näčä elig ulušug

$07 \quad[\quad$ ay $] \lg \left[k_{1}\right] \ln \ln _{\text {ch }} \operatorname{lig}_{1}$

08 iš-lärtä etilmäk [üzä] ü[č yavla]k

09 yol-larka ${ }^{29}$ tüšsär $\mathrm{t}[\quad$ üč $]$

10 ärdini tıltagınta ärür : amtı [ ]

11 [üč] ärdini-lär-kä tayanmak [üzä] üč

$12 \mathrm{y}(\mathrm{a})$ vlak yol-ta [bar]guluk ayıg [kılınč]

$13-1 \mathrm{~m}(1) z n ı$ arıtmıšım(1)z ketärmišim(i)z k(ä)rgäk

14 anta näčä agır ulug buyan-lıg iš-lär

15 -ig išlämišim(i)z ymä bar : näčä bar

\section{Übersetzung}

01 (...) haben wir [ ] verloren. [ ]. 02-03 Es gibt nichts Besseres als das Hören der W[orte] des Göttergotts Buddha. 03-10 I[hr] haltet [ ], an welchen Ort man auch abgleitet, wird man, wenn man [] sich auf den [ ] Ort stützt,

27 Orzech, 1998: 96.

28 Unklar, welche Seite recto ist und welche verso.

${ }^{29}$ Vielleicht auch ywl l'rt', falls das <t> sehr klein ausgefallen ist. 


\section{J(O)}

bestehen; wenn man durch das Verstricktsein in Sachen das Reich [schädigender] [schlech]ter Taten auf die dr[ei schlecht]en Wege fällt, ist es wegen [der mißachteten (?) drei] Juwelen.

10-13 Jetzt [ ] sollen wir durch das Sich-Stützen auf die drei Juwelen unsere schlechten Taten, [durch die man gelangt] auf die drei schlechten Wege, reinigen und vernichten. 14-15 Wie sehr wir Taten mit sehr großen Verdiensten verrichten, so sehr (...).

\section{Verso (?)}

16 [ ] kurug-1[ar ]

17 [ isig] öznün yitlinmäki [yavrımakı]

18 [bo]l[u]r ärsär : ančulayu [ymä el-niy]

19 uluš-nun yavrımakı [yitlinmäki ärip]

20 el i-ä-[s]i-nin y[itlinmäki yavrı]

21 -makı bolur : bo ok tö[rt ulugka tükäl]

22 -lig ät'öznüy üklimä[ki asılmakı]

23 üzä nätäg isig öznüy [a]sı1[makı]

24 üklitmäki bolur ärsär : anı täg

25 [ el-nin u]l[u]š-nuy üklimäki

26 [asıl]makı üzä el i-ä-läri-ni[ๆ]

27 tirilmäki yašamakı bolur tep : yänä

28 kanımz t(ä)yri burhan y(a)rlıkamıšı bar :

29 kayu eliglär bäglär mänin šazınımın

30 kigür[ü]p nomlug isig özümin ulagay

\section{Übersetzung}

(...) [ ] die Leeren [], was das Verschwinden und Schwachwerden des Lebens betrifft, ist es so wie das Schwachwerden und Verschwinden des Reichs und das Schwachwerden und Verschwinden des Landesherrn. So wie der mit den Vier Großen ausgestattete Körper wächst und zunimmt und so das Leben wächst 
und zunimmt, so ist es das Zunehmen und Anwachsen des Reiches und das Zunehmen und Anwachsen des Landesherrn. Ferner hat unser Vater, der göttliche Buddha, gesagt: "Welche Könige oder Fürsten meine Disziplin einführen, die werden mein Lehr-Leben verlängern!"

Dieser Text, insbesondere der letzte hervorgehobene Satz, sieht aus wie eine Replik auf die große Sorge des Buddha, die er in einem Gespräch mit König Prasenajit zum Ausdruck bringt ${ }^{30}$, wenn er beschreibt, daß nach seinem Nirvāna die Wesen in die buddhalose Zeit der ,Fünf Trübungen“ und die Lehren in Verfall geraten, wodurch auch die Staaten zugrundegehen. Komischerweise ist die Antwort Prasenajits nur, wie man denn das Sütra nennen solle, woraufhin der Buddha antwortet:

"Great king! The merit of this Prajñāpāramitā is like the void, it cannot be fathomed. If you receive and keep, read, and recite it, the merit obtained will be able to protect Humane Kings and even all beings, like walls, yea, like the walls of a city. Therefore you and the others should receive and keep it." ${ }^{31}$

Auch eine Passage im Mahāparinirvāna mahāsūtra beleuchtet diese Zukunftssorge:

\begin{abstract}
"O good man! The same is the case with the Tathagata. He views those who transgress Dharma as he views his only son. The Tathagata now entrusts unsurpassed Wonderful Dharma to the hands of kings, ministers, prime ministers, bhiksus, bhiksunis, upasakas and upasikas. All of these kings and the four classes of the Buddhist Sangha will encourage those who practise the Buddhist teaching and enable them increasingly to observe the moral precepts, practise meditation and wisdom. If there are any who miscarry these three phases [aspects] of Dharma and if there are those who are indolent and who break the moral precepts, the kings, ministers, and the four classes of the Buddhist Sangha will work hard and remould such people." 32
\end{abstract}

Natürlich ist das Thema damit nicht erschöpft. Die vier Begegnungen der Buddhas gehören zu festen Bestandteilen der buddhistischen Tradition, der sich auch die uigurischen Buddhisten verpflichtet fühlten. Wie deutlich geworden ist, haben sie dieses große Thema mit Tiefe und Vielfalt immer wieder aufgegriffen. Man darf hoffen, daß durch die Erschließung neuer Fragmente auch weitere Aspekte beleuchtet werden können, wobei die Beziehungen zwischen den vier

\footnotetext{
30 T 246, Übersetzung von Orzech, 1998: 271-274.

31 Orzech, 1998: 274.

32 Yamamoto, 2007: 38.
} 


\section{J(৫)}

Begegnungen und den vier Wahrheiten als ein Komplex näher erforscht werden sollten.

\section{Literaturverzeichnis}

Gabain, A. von (1967). Die Drucke der Turfan-Sammlung. Sitzungsberichte der Deutschen Akademie der Wissenschaften zu Berlin. (Klasse für Sprachen, Literatur und Kunst, 1967: 1).

Inagaki, H. (1994). The Three Pure Land Sutras, a Study and Translation from Chinese. In collaboration with $\mathrm{H}$. Stewart. Nagata Bunshodo.

Kara, G. (2009). Dictionary of Sonom Gara's Erdeni-yin Sang. A Middle Mongol Version of the Tibetan Sa skya Legs bshad. Mongol-English-Tibetan. Brill.

Kirfel, W. (1920). Die Kosmographie der Inder nach den Quellen dargestellt. Olms.

Meier, F. J. [1988]. Die Mythologie des chinesischen Buddhismus. Klett-Cotta [1988]. (Wörterbuch der Mythologie. I. Abteilung Die alten Kulturvölker 23. + 24. Lieferung).

Monier-Williams, M. (1899). A Sanskrit-English Dictionary, Etymologically and Philologically Arranged with Special Reference to Cognate Indo-European Languages. Motilal Banarsidass Publishing House.

Nadeljaev, V. M. et al. (1969). Drevnetyurkskiy Slovar'. İzdatel'stvo „Nauka“ Leningradskoye Otdeleniye Leningrad.

Nattier, J. (1991). Once upon a future time. Studies in a prophecy of decline. Asian Humanities Press.

Nobel, J. (1958). Suvarnaprabhāsottamasūtra, das Goldglanz-Sūtra, ein Sanskrittext des Mahāyāna-Buddhismus, I-Tsing's chinesische Version und ihre tibetische Übersetzung I-II. Brill.

Orzech, C. D. (1998). Politics and Transcendent Wisdom: The Scripture for Humane Kings in the Creation of Chinese Buddhism. Pennsylvania State University.

Radloff, W. (1917). Uigurisches Wörterbuch. ${ }^{33}$

Soothill, W. E. \& Hodous, L. (1937). A Dictionary of Chinese Buddhist Terms with Sanskrit and English Equivalents and a Sanskrit-Pali Index. Kegan Paul, Trench, Trübner \& Co.

33 Details nach Nadeljaev et al., 1969: III. Lemmata von /a/, /ä/, /e/, /1/ und /i/ wurden in 208 Spalten gedruckt. Das komplette Manuskript wird im Archiv der Russischen Akademie aufbewahrt: Fonds 177, Abt. 1, Nr. 80, 82, 90. 


\section{J曰)}

Wilkens, J. (2016). Buddhistische Erzählungen aus dem alten Zentralasien. Edition der altuigurischen Daśakarmapathāvadānamālā I-III. Berliner Turfantexte 37. Brepols Publishers.

Wojtilla, G. (1973). Indian Precious Stones in the Ancient East and West. Acta Orientalia Academiae Scientiarum Hungaricae, 27(2), 211-224.

Yamamoto, K. (2007). The Mahayana Mahaparinirvana Sutra. (Internet-Version).

Zieme, P. (2020). Minora fragmenta veterorvm vigvrorvm. Berliner Turfantexte 47. Brepols Publishers. 